\title{
Observation of Bernstein Wave Propagating Obliquely to the Magnetic Field in a Streaming Plasma
}

\author{
T. Idehara*, M. Takeda* and Y. Ishida*
}

(Received March 12, 1973)

\begin{abstract}
Bernstein wave propagating obliquely to the magnetic field is excited by a coaxial antenna in a streaming plasma and detected by the interferometer system, which shows that the dispersion relation for the wave is consistent with the theoretical result. The other wave-like signal with shorter 'wavelength' is observed only on the downstream of the exciting antenna and its 'phase velocity' is approximately equal to the velocity of plasma stream calculated from the Doppler shifts of Bernstein waves on both up- and down-streams, which may show that the signal does not propagate as the wave but floats down the plasma stream as the modulation of plasma particles.
\end{abstract}

\section{Introduction}

It is predicted by Bernstein ${ }^{1)}$ that the electrostatic wave propagating perpendicularly to the magnetic field has many branches near the harmonics of cyclotron frequency and can propagate without Landau damping, because the resonant particle for the wave does not exist. Then, the wave was observed in the dc discharge plasma in low pressure rare gases ${ }^{2)}$ and in the electron beam-plasma system"3, which is consistent with the theoretical consideration ${ }^{4}$ that the superthermal electron can excite the wave. However, as shown by Tataronis and Crawford ${ }^{5)}$, the wave propagating obliquely to the magnetic field does heavily Landau-damp in thermal equilibrium, so that the wave can propagate only almost perpendicularly to the magnetic field. Perpendicularly propagating Bernstein wave has been observed by several authors ${ }^{6)}$, who show that its dispersion relation agrees with the theoretical results $^{5)}$ completely. In this letter, we report the observation of Bernstein wave propagating obliquely to the magnetic field in the streaming plasma and show that the observed dispersion relation is consistent with the theoretical consideration ${ }^{5}$.

\section{Experimental apparatus}

The plasma is produced in the TP-D type device ${ }^{\eta)}$ as shown in Fig. 1, which consists of the discharge region and the difiused region of plasma, both regions

* Department of Applied Physics 
being connected by the orifice (200 $\mathrm{mm}$ in length and $10 \mathrm{~mm}$ in diameter) and the pressures of the former and the latter being maintained at about $1.5 \times 10^{-2}$ Torr and $7.5 \times 10^{-4}$ Torr respectively, by using the method of differential pumping. The plasma is jetted through the orifice from the hole $(8 \mathrm{~mm}$ in diameter) in the center of anode, flows along the line of magnetic force into the diffused region of plasma, which is made of pyrex glass tube $(720 \mathrm{~mm}$ in length and $95 \mathrm{~mm}$ in diameter) and is ended at the collector of the same voltage as the anode, and is supported on the axis of the glass tube by the uniform magnetic field whose intensity is 60 gauss (the electron cyclotron frequency $\omega_{\mathrm{c}} / 2 \pi$ is $168 \mathrm{MHz}$ ). The plasma density profile in the radial direction is shown in Fig. 2 (b) and that in the axial direction is uniform within about 5 percent. When the maintenance voltage of discharge $V_{d}$ is about 100 volt and discharge current $I_{d}$ is $4.5 \mathrm{~mA}$, the plasma density $n_{v}$ at the center of tube and the electron temperature $\mathrm{T}_{\mathrm{e}}$ is about $1.5 \times 10^{9} \mathrm{~cm}^{-3}$ and $7.8 \mathrm{eV}$ respectively in diffused region of plasma.

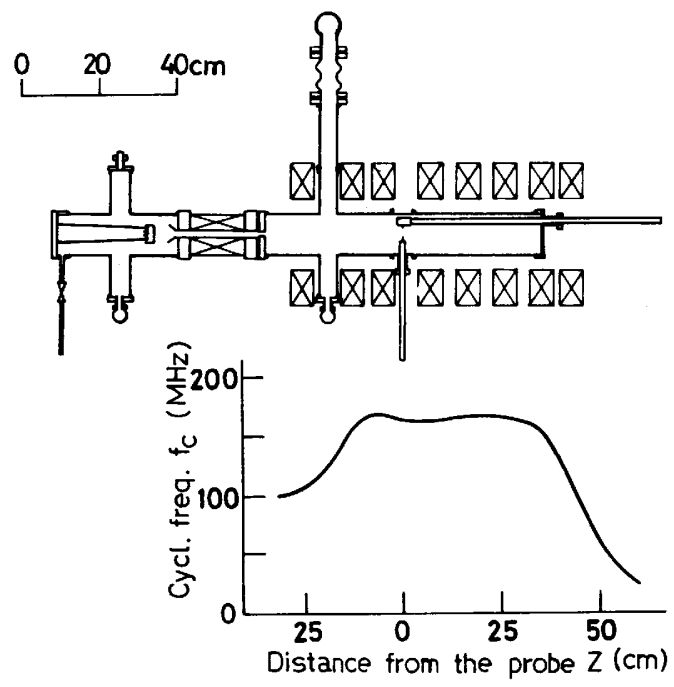

Fig. 1 The experimental device and the magnetic field strength as a function of the axial distance from the exciting antena $z$.

\section{Experimental results and discussions}

The Bernstein wave is excited by the coaxial antenna inserted radially at the axial position of $390 \mathrm{~mm}$ from the anode and detected by two coaxial antennae movable in the radial and axial directions. By using the interferometer system, the propagating wave patterns are drawn on $\mathrm{XY}$ recorder as the functions of axial distance $z$ and radial distance $r$ from the exciting antenna, as shown in Fig. 2 . (a) and (b). It must be noted in Fig. 2 (a), that the wavelength in the region of downstream is longer than that of upstream, the both waves being damped rapidly by Landau damping, and the other wave-like signal of shorter 'wavelength' is observed only in the region of downstream. In Table 1 , the wave number components along the direction of axis $\left(\mathrm{k} \|_{\|}\right)$and radius $\left(\mathrm{k}_{\perp}\right)$ are calculated from the observed wave patterns, which shows that the observed Bernstein wave propagates at an angle $\theta$ of about $85^{\circ}$ from the line of magnetic force.

In Fig. 3 are shown the dispersion relations of observed Bernstein waves in upstream and downstream, which are slightly different each other due to the Doppler effect in streaming plasma. From the difference of phase velocities in the both regions, the streaming velocity $V_{s}$ of plasma is calculated and shown in Table 1. The result indicates that $V_{s}$ is $4.1 \times 10^{8} \mathrm{~cm} / \mathrm{sec}$ within the observation error of 18 


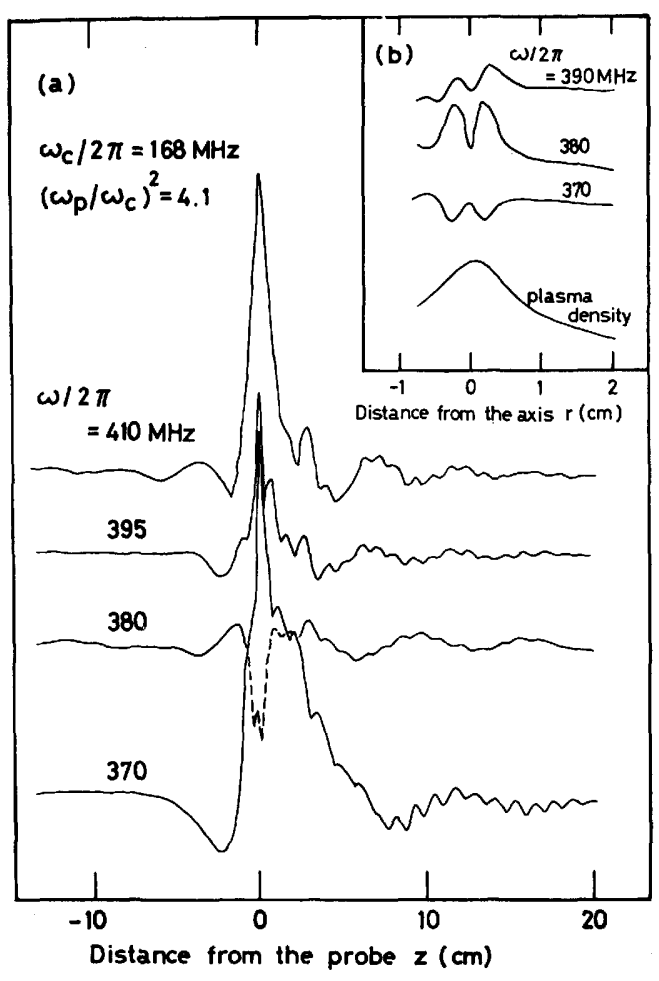

Fig. 2 (a) The propagating wave patterns as functions of the axial distance from the exciting antenna $z$. The anode is situated at $z=-39 \mathrm{~cm}$, so that the plasma is streaming from the left hand side to the right hand side. (b) The propagating wave patterns and the plasma density profile as functions of the radial distance from the axis $r$.

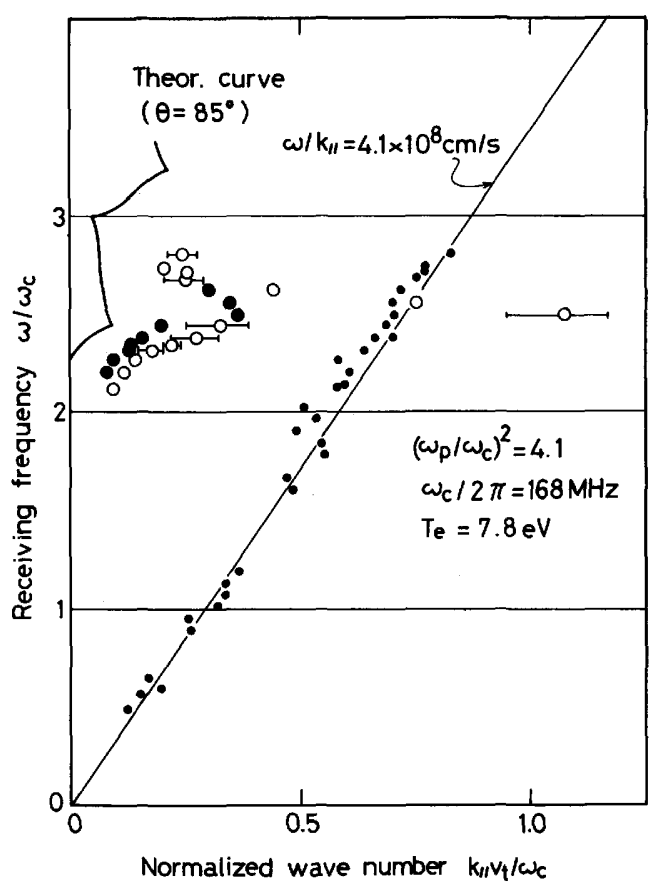

Fig. 3 The dispersion relation calculated from the wave patterns and the theoretical curve for the corresponding experimental conditions. hollow circle; Bernstein wave in the region of upstream. larger solid circle ; Bernstein wave in the region of downstream. smaller solid circle; the wave-like signal with shorter 'wavelength' observed only in the region of downstream.

Table 1 The relation among the frequency $(\omega / 2 \pi)$, the wave number components $\left(\mathrm{k}_{\|}, \mathrm{k}_{\perp}\right)$ and the streaming velocity $\left(V_{s}\right)$ calculated from the difference of the phase velocities in the regions of $\mathrm{up}^{-}$and down-streams.

\begin{tabular}{|c|c|c|c|c|c|}
\hline $\begin{array}{l}\omega / 2 \pi \\
(\mathrm{MHz})\end{array}$ & $\mathrm{k}_{\perp}-\frac{\mathrm{v}_{\mathrm{t}}}{\omega_{\mathrm{c}}}$ & $\mathrm{k}_{\|} \frac{\mathrm{V}_{1}}{\omega_{\mathrm{c}}}$ & $\theta=(\hat{\mathrm{kB}})$ & $\begin{array}{c}\omega / \mathrm{k}_{\|} \\
\left(\times 10^{8} \mathrm{~cm} / \mathrm{s}\right)\end{array}$ & $\frac{\mathrm{V}_{\mathrm{s}}}{\left(\times 10^{8} \mathrm{~cm} / \mathrm{s}\right)}$ \\
\hline \multirow[b]{2}{*}{390} & \multirow[b]{2}{*}{1.50} & 0.181 & $83.1^{\circ}$ & 15.13 (upstream) & \multirow{2}{*}{4.87} \\
\hline & & 0.133 & $84.8^{\circ}$ & 20.60 (downstream) & \\
\hline \multirow{2}{*}{380} & \multirow{2}{*}{1.68} & 0.144 & $85.1^{\circ}$ & 18.62 (upstream) & \multirow{2}{*}{4.05} \\
\hline & & 0.100 & $86.6^{\circ}$ & 26.71 (downstreaj) & \\
\hline \multirow{2}{*}{370} & \multirow{2}{*}{1.70} & 0.118 & $86.0^{\circ}$ & 22.05 (upstream) & \multirow{2}{*}{3.94} \\
\hline & & 0.087 & $87.1^{\circ}$ & 29.93 (downstream) & \\
\hline
\end{tabular}


percent. On the other hand, the wave-like signals with shorter 'wavelength' observed only on the downstream are plotted by smaller circles in Fig. 3, which are distributed near the straight line of $\omega / \mathrm{k}_{\|}=4.1 \times 10^{8} \mathrm{~cm} / \mathrm{sec}\left(=\mathrm{V}_{\mathrm{s}}\right)$. The fact mentioned above does support the consideration that the plasma is streaming with the velocity $V_{s}$ and the latter wave-like signal may not be the propagating wave but only the modulation of plasma particles floating with the plasma stream.

The theoretical dispersion relation curve corresponding to our experimental conditions $\left(\omega_{p}{ }^{2} / \omega_{c}{ }^{2}=4.1\right.$ and $\left.\theta=(\hat{\mathrm{kB}})=85^{\circ}\right)$ is shown with the observed points in Fig. 3 The both results are in good agreement qualitatively. It may be considered that the quantitative disagreement results from the overestimate in electron temperature $T_{e}$ observed by the Langmuir probe method, because the plasma is streaming with the velocity $\mathrm{V}_{\mathrm{s}}$

The damping factor (the imaginary part of $\mathrm{k} \|$ ) was so large that it could not be measured. In conclusion, the Doppler shifted Bernstein wave propagating obliquely to the magnetic field is observed in the streaming plasma and its dispersion relation is in agreement with the theoretical curve qualitatively.

\section{Acknowled gement}

The authors wish to express their thanks to Mr. I. Kasuga for his kindness to prepare the glass tubes. This work was partially supported by the Grant-in-Aid from the Ministry of Education.

\section{References}

1) I. B. Bernstein Phys. Rev. 10910 (1958).

2) G. Landauer, J. Nucl. Energy, Pt. C, 4395 (1962); K. Mitani, H. Kubo and S. Tanaka, J. Phys. Soc. Japan 19211 (1964); G. Bekefi, J. D. Coccoli, E. B. Hooper and S. J. Buchsbaum, Phys. Rev. Letters 96 (1962) ; C. D. Lustig. Appl. Phys. Lettes 4194 (1 964).

3) M. Seidl and P. Sunka, Nucl. Fusion 7237 (1967) ; T. Idehara, K. Ohkubo and S. Tanaka, J. Phys. Soc. Japan 27187 (1969) ; B. R. Kusse and A. Bers, Phys. Fluids 135372 (1970).

4) E. Canobbio and R. Croci, J. Nucl. Energy Pt. C, 9549 (1966).

5) J. A. Tataronis and F. W. Crawford, J. Plasma Physics 4231 (1970) and 4249 (1970).

6) S. Gruber and G. Bekefi, Phys. Fluids 11122 (1968; F. Leuterer, Plasma Physics 11 615 (1969).

7) M. Ohtsuka, K. Takayama et al., Proc. 7th Intern. Conf. on Ionization Phnomena in Gases, Beograde, August 1965 . 